\title{
How to Model an Ising Ferroelectric System: Case of the Investigation of the Dielectrics Properties of a Nano-Octahedral Ferroelectric System
}

\author{
Alioune Aidara Diouf ${ }^{1,2, ~ *, ~ B a s s i r o u ~ L o ~}{ }^{1}$, Abdoulaye Ndiaye Dione ${ }^{1}$, Cheikh Birahim Ndao ${ }^{1}$, \\ Aboubaker Chedikh Béye ${ }^{1}$ \\ ${ }^{1}$ Faculty of Sciences \& Techniques, Cheikh Anta Diop University, Dakar, Senegal \\ ${ }^{2}$ Department of Nanoscience \& Nanotechnology Research, Dakar American University of Science \& Technology, Somone, Senegal \\ Email address: \\ aliouneaidara.diouf@ucad.edu.sn (A. A. Diouf) \\ *Corresponding author
}

\section{To cite this article:}

Alioune Aidara Diouf, Bassirou Lo, Abdoulaye Ndiaye Dione, Cheikh Birahim Ndao, Aboubaker Chedikh Béye. How to Model an Ising Ferroelectric System: Case of the Investigation of the Dielectrics Properties of a Nano-Octahedral Ferroelectric System. Communications. Vol. 5, No. 5, 2017, pp. 51-57. doi: 10.11648/j.com.20170505.11

Received: January 5, 2018; Accepted: January 15, 2018; Published: January 31, 2018

\begin{abstract}
The effective field theory within a probability distribution technique that accounts for the self-spin correlation functions is used to investigate the dielectrics properties of a nano-octahedral system described by the spin $\mathrm{S}=1 / 2 \mathrm{Ising}$ model. The thermal behavior of the polarizations, susceptibilities, and the hysteresis loops are examined in details and even the details to model a ferroelectric system.
\end{abstract}

Keywords: Transverse Ising Model, Ferroelectric, Dirac, Hamiltonian, Polarization, Hysteresis, Susceptibility

\section{Introduction}

In recent years, the investigations on the ferroelectrics (FE) properties present a particular interest for the scientists. Grace to their importance in the fields of microelectronics and optoelectronics the ferroelectrics systems play a precious role in the new technology. Several authors already worked experimentally [1-5] and theoretically [6-15] in the ferroelectrics fields. To model ferroelectrics phenomenon the transverse Ising model (TIM) within the effective field theory (EFT) are well used by the authors [16]. Gennes and al. [17] were the first to apply the TIM and Blinc and al. [18] used it to describe order-disorder type ferroelectrics. Several TIM have been applied to investigate various static and dynamic properties of ferroelectrics [19-34]. All these researches show the simplicity to use TIM. The rigorous results which provide TIM to interpret the theoretical investigations are looking for by the scientists. Reason why for our study we use TIM to model the dielectric properties of a nano-octahedral ferroelectric structure. The objective in the present paper is to present the steps to model a ferroelectric system by using the
TIM and predict from a nano-octahedral system the dielectric properties of a sample considered in a large number with a comparative investigation of the influence of the transverse field and the exchange interaction on the dielectrics properties. One will investigate the polarizations, longitudinal susceptibilities and the hysteresis loops of a ferroelectric nano-octahedral system spin $S=1 / 2$ within the framework of the effective field theory with probability distribution technique. In the Section 2, one will present the model and formalism used for the transverse Ising model. Furthermore the effects of the external electric field, temperature, transverse field and exchange interaction on the dielectric properties are well discussed in detail in the Section 3.

\section{Model and Formalism}

Consider a nano-octahedral structure defined by the following structure. 


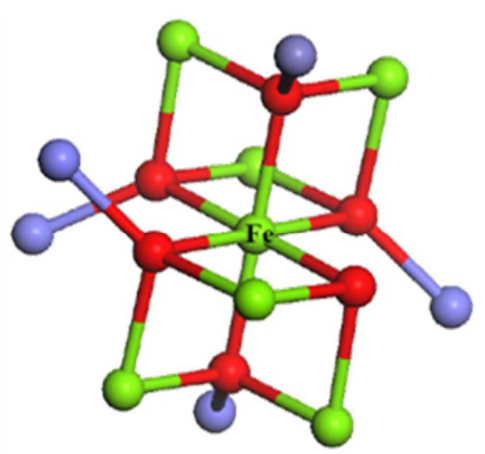

Figure 1. Nano-octahedral structure of $\mathrm{Fe}_{2} \mathrm{O}_{3}$.

The atom in the center labelled $(\mathrm{Fe})$ of the structure plays the role of the central spin and the red atom play the role of the nearest neighbors. The method to establish the order moment is the same than [35]. Therefore the transverse Ising model used to study our ferroelectric system is defined by:

$$
H=-\sum_{(i j)} J_{i j} S_{j z} S_{i z}-\Omega \sum_{i} S_{i x}-2 \mu E \sum_{i} S_{i z}
$$

where $H=-A S_{i z}+B S_{i x}$

$$
A=\sum_{(i j)} J_{i j} S_{j z}+2 \mu E \text { and } B=-\Omega .
$$

With $J_{i j}$ the exchange interaction, $S_{i z}$ and $S_{i x}$ the components following $(\mathrm{Oz})$ and $(\mathrm{Ox})$ of the spin $\mathrm{S}=1 / 2$, E the electric field, $\mu$ the dipole moment, the sum $\sum_{(i j)}$ run over all sites and $\Omega$ the transversal field.

$S_{i z}$ and $S_{i x}$ are represented by the following matrix:

$$
S_{i z}=\left(\begin{array}{cc}
\frac{1}{2} & 0 \\
0 & -\frac{1}{2}
\end{array}\right) \text { and } S_{i x}=\left(\begin{array}{cc}
0 & \frac{1}{2} \\
\frac{1}{2} & 0
\end{array}\right)
$$

To calculate the different averages, one must find the eigenvalues and the associated vectors of the Hamiltonian $\mathrm{H}$ [36], which is represented by the following matrix:

$$
H=\left(\begin{array}{cc}
\frac{1}{2} & \frac{1}{2} \\
\frac{1}{2} & -\frac{1}{2}
\end{array}\right)
$$

and the corresponding vectors are given by:

$$
\left\{\begin{array}{l}
E_{1} \longrightarrow\left|\Psi_{1}\right\rangle=a_{1}\left|\frac{1}{2}\right\rangle+b_{1}\left|\frac{1}{2}\right\rangle \\
E_{2} \longrightarrow\left|\Psi_{2}\right\rangle=a_{2}\left|\frac{1}{2}\right\rangle+b_{2}\left|-\frac{1}{2}\right\rangle
\end{array}\right.
$$

the quantities $\left|\frac{1}{2}\right\rangle$ and $\left|-\frac{1}{2}\right\rangle$ are the associated vectors of $S_{z}$.

The coefficients $a_{k}, b_{k}(k=1,2)$ can be calculated from the following equations [37]:

$$
H\left|\Psi_{k}\right\rangle=E_{k}\left|\Psi_{k}\right\rangle
$$

and one has

$$
\left(\begin{array}{cc}
\frac{A}{2} & \frac{B}{2} \\
\frac{B}{2} & -\frac{A}{2}
\end{array}\right)\left(\begin{array}{l}
a_{k} \\
b_{k}
\end{array}\right)=\left(\begin{array}{l}
a_{k} \\
b_{k}
\end{array}\right)\left|\Psi_{k}\right\rangle
$$

Taking account of the conditions of the following normalization of the states:

$$
\left\langle\Psi_{k} \mid \Psi_{k}\right\rangle=a_{k}^{2}+b_{k}^{2}=1
$$

Thus, one has:

$$
a_{k}^{2}=\frac{B^{2}}{\left(A-2 E_{k}\right)^{2}+B^{2}} b_{k}^{2}=\frac{\left(A-2 E_{k}\right)^{2}}{\left(A-2 E_{k}\right)^{2}+B^{2}}
$$

From statistics of spin systems, for the operators $S_{i r}^{\alpha}$ at site i, one has:

$$
\left\langle S_{i r}^{\alpha}\right\rangle=\left\langle\frac{\operatorname{Tr}\left(S_{i r}^{\alpha} \exp (-\beta H)\right.}{\operatorname{Tr}(\exp (-\beta H)}\right\rangle
$$

Where the angular bracket $\langle\ldots\rangle$ denotes a canonical thermal average, $\beta=1 / \mathrm{k}_{\mathrm{B}}$. T, T is the temperature and $\alpha$ is the number of order moment. If the exchange interactions are restricted to nearest-neighbors interactions only, the evaluation of the inner traces over selected spins in the last equations yields is

$$
\left\langle S_{i r}^{\alpha}\right\rangle=\left\langle F_{\alpha}(A, B)\right\rangle
$$

Where $\left\langle F_{\alpha}(A, B)\right\rangle=\frac{A}{\sqrt{A^{2}+B^{2}}} \tanh \left(\frac{1}{2} \beta \sqrt{A^{2}+B^{2}}\right) \quad$ if $\quad r=z$ : Longitudinal and $\left\langle F_{\alpha}(A, B)\right\rangle=\frac{B}{\sqrt{A^{2}+B^{2}}} \tanh \left(\frac{1}{2} \beta \sqrt{A^{2}+B^{2}}\right)$ if $r=x$ : Transversal

The above thermal averages are valid for a fixed spatial configuration. The step is to carry out the configurational averaging to be noted $\langle\ldots\rangle_{r}$, the quantities of interest are the polarization $P^{\alpha}$ defined by:

$$
P^{\alpha}=\left\langle\left\langle S_{i r}^{\alpha}\right\rangle\right\rangle=\left\langle\left\langle F_{\alpha}(A, B)\right\rangle\right\rangle
$$

$P^{\alpha}$ is function depending of $\sum_{i=0}^{N} S_{i r}$ like: $F\left(\sum_{i=0}^{N} S_{i r}\right)$. One introduces the Dirac delta function: 


$$
\begin{aligned}
& \delta\left(y_{1}-\sum_{i=0}^{N} S_{i r}\right)=\int \frac{d \lambda_{1}}{2 \pi} e^{i\left[y_{1}-\sum_{i=0}^{N} S_{i r}\right]} \\
& \delta\left(y_{1}-\sum_{i=0}^{N} S_{i r}\right)=\int \frac{d \lambda_{1}}{2 \pi} e^{-i \lambda_{1} S_{i r}}
\end{aligned}
$$

One has:

$$
\begin{gathered}
\left\langle F\left(\sum_{i=0}^{N} S_{i r}\right)\right\rangle=\int d y_{1}\left(y_{1}-\sum_{i=0}^{N} S_{i r}\right) F\left(y_{1}\right) \\
F\left(\sum_{i=0}^{N} S_{i r}\right)=\int d y_{1} F\left(y_{1}\right) \int \frac{d \lambda_{1}}{2 \pi} \prod_{i=1}^{N} e^{-i \lambda_{1} S_{i r}}
\end{gathered}
$$

In the approximation of Zernike decoupling of the multiple correlation, one has:

$$
\left\langle F\left(\sum_{i=0}^{N} S_{i r}\right)\right\rangle=\int d y_{1} F\left(y_{1}\right) \int \frac{d \lambda_{1}}{2 \pi} \prod_{i=1}^{N}\left\langle e^{-i \lambda_{1} S_{i r}}\right\rangle
$$

To perform the configurational averaging on the right-hand side of the last equation, one uses the probability distribution method based on the use of generalized Van der Wander identities [38]. If this is done one has:

$$
\prod_{i=1}^{N}\left\langle e^{-i \lambda_{1} S_{i r}}\right\rangle=\prod_{i=1}^{N}\left(\sum_{S_{i r}=-1 / 2}^{1 / 2} P\left(S_{i r}\right) e^{-i \lambda_{1} S_{i r}}\right)
$$

Where the distribution functions $V\left(S_{i r}\right)$ are given by:

$$
V\left(S_{i r}\right)=\frac{1}{2}\left[\left(1-2 p_{z}\right) \delta_{S_{i r,-1 / 2}}+\left(1+2 p_{z}\right) \delta_{S_{i r, 1 / 2}}\right]
$$

Taking into account relations above, one gets the following relations for the order moments:

$$
\begin{aligned}
& P^{\alpha}=\left\langle\left\langle F_{\alpha}(A, B)\right\rangle\right\rangle=2^{-(N+1)} \sum_{\mu_{1}=0}^{N} C_{\mu_{1}}^{N}\left(1+p_{z}\right)^{N-\mu_{1}} \\
& \left(1-p_{z}\right)^{\mu_{1}} \operatorname{th}\left(J_{\alpha}\left(\left(N-2 * \mu_{1}\right)+2 \mu E\right)\right)
\end{aligned}
$$

In this equation, $\mathrm{N}$ represent the coordination number and $C_{k}^{n}$ is the binomial coefficients, $C_{k}^{n}=\frac{n !}{k !(n-k) !}$. One has the self-consistent equations for the order moments $P^{\alpha}$ with the nano-octahedral ferroelectric system used in this paper, which can be solved directly by numerical iteration without any further algebraic manipulations. Then the average total polarization is given by:

$$
P=\frac{\sum_{i=1}^{n} P_{i}^{z}}{N}
$$

With $\mathrm{N}$ the total number of spin in the nano-octahedral ferroelectric structure represent in the Figure 1.

The longitudinal susceptibility for the system can be determined from the following relation:

$$
\chi=\frac{\delta P}{\left.\delta E\right|_{E=0}}
$$

The nano-octahedral ferroelectric system (Figure 1) is used to study the dielectric properties. Instead of our method in [35], taking into account $\mathrm{J}_{\mathrm{S}}$ which represent the exchange interaction of shell, $\mathrm{J}_{\mathrm{C}-\mathrm{S}}$ the exchange interaction of coreshell, $\mathrm{J}_{\mathrm{C}}$ the exchange interaction of core-core, in this paper we neglected the interaction between atom of shell and we consider only the exchange interaction $\mathrm{J}_{\mathrm{C}-\mathrm{S}}$ between spin of the center and the spin of the surface.

\section{Results and Discussions}

Polarizations - The enhancement of the exchange interaction study in the Figure 2 shows the evolution of the polarization according to the temperature. One observes that the exchanges interactions $\mathrm{J}_{\mathrm{c}-\mathrm{s}}$ for 1.0, 1.5 and 2.0, 2.5 give, respectively, the same behavior about the polarization with the different transitions temperatures $\mathrm{k}_{\mathrm{B}}$. $\mathrm{T}_{\text {Curie }}=1.20\left(\mathrm{~J}_{\mathrm{c}-\mathrm{s}}=\right.$ 1.0 and 1.5) and $\mathrm{k}_{\mathrm{B}}$. $\mathrm{T}_{\text {Curie }}=2.50\left(\mathrm{~J}_{\mathrm{c}-\mathrm{s}}=2.0\right.$ and 2.5) and for $\mathrm{J}_{\mathrm{c}-\mathrm{s}}=3.0, \mathrm{k}_{\mathrm{B}} . \mathrm{T}_{\text {Curie }}=3$. One can interpret the shape of the curves and the physical phenomenon by the phase transition from the ferroelectric effect to the para-electric. One already observed these behaviors in the authors [16] who investigated the ferroelectric thin film with the Effective field theory. The transition temperature called Curie temperature defined the temperature where the nano-octahedral ferroelectric system pass from an ordered structure (Ferro) to a disordered structure (para) of the spins. In the Figure 3, one did not observe the same phenomenon, even if one saw the same ferroelectric behavior about the evolution of the polarization according to the evolution of the transverse field $(\Omega)$. One notes here more the transverse field increases more the polarization and the critical temperature decrease (for $\Omega=1.0$, $\mathrm{k}_{\mathrm{B}}$. $\mathrm{T}_{\text {Curie }}=1.20$, for $\Omega=1.5, \mathrm{k}_{\mathrm{B}} . \mathrm{T}_{\text {Curie }}=1.08$, for $\Omega=2, \mathrm{k}_{\mathrm{B}}$. $\mathrm{T}_{\text {Curie }}=0.88$, for $\Omega=2.5$ and $\left.3, \mathrm{p}(\mathrm{T})=0.0\right)$. One can interpret the phenomenon by a perturbation of the transverse field, the influence of the transverse field reduces until cancel the order of the spins linked by the dipole moment which is a function of the exchange interaction responsible of the ferroelectric state of the system that is why we observe from $\Omega=1.0$ to 3 the values of the polarization move from $\mathrm{p}(\mathrm{T})=0.47$ to 0.0 . In the Figure 4, with the evolution of the polarization according to the enhancement of the electric field (E). One observe that the polarization increases when the electric field increases but one don't observe a critical temperature which could define the transition ferro-paraelectric. One can interpret this phenomenon by the fact that because of the non-zero applied field, the electric field create a perturbation of the unit cell and support the augmentation of the polarization instead of the phase transition and authors [39] already noted this kind of behavior with maghemite nanoparticles $\gamma-\mathrm{F}_{2} \mathrm{O}_{3}$ and E. K. Tan and al. [7] observed this behaviors with a ferroelectric thin film and interpret the phenomenon by the size effect. Reason why there is no longer phase transition therefore the polarization simply decreases monotonically and the free 
energy approaches zero only asymptotically.

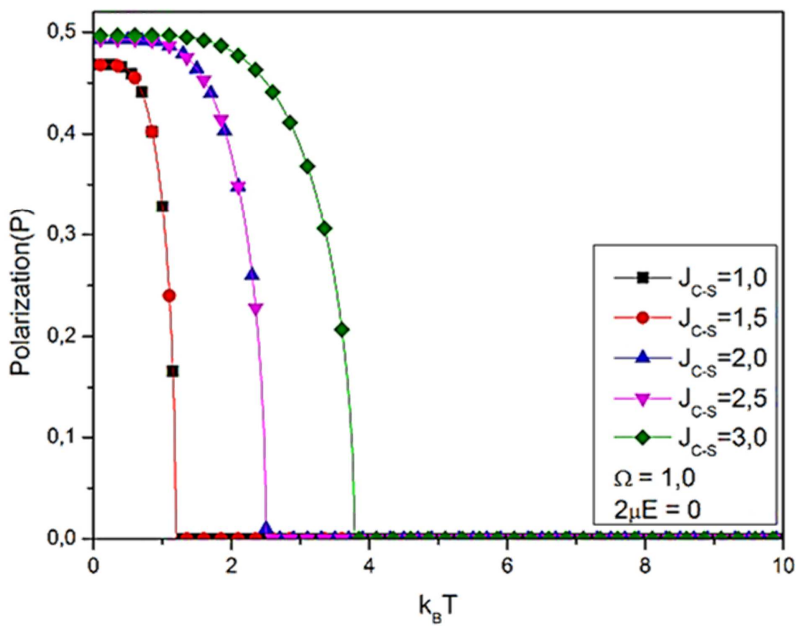

Figure 2. Polarization according to the evolution of the exchange interaction $\left(J_{C-S}\right)$.

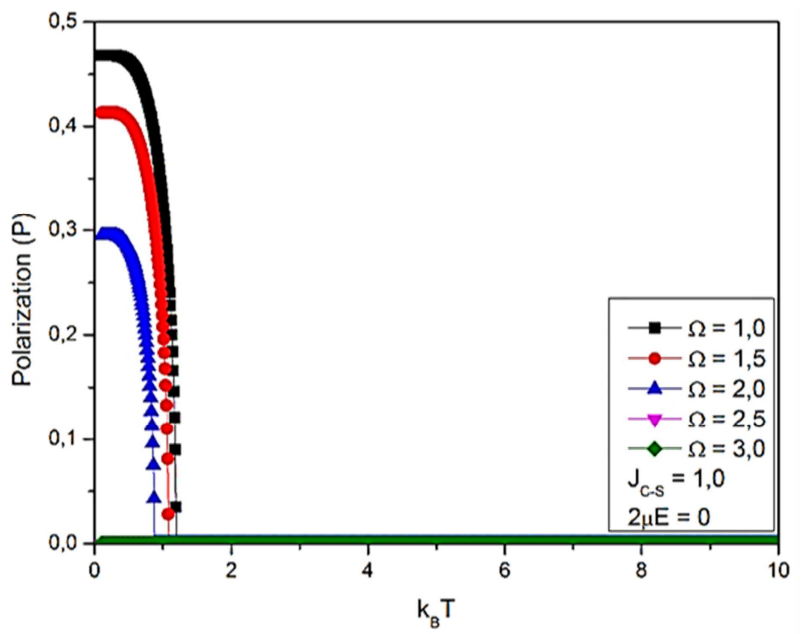

Figure 3. Polarization according to the evolution of the transverse field ( $\Omega$ ).

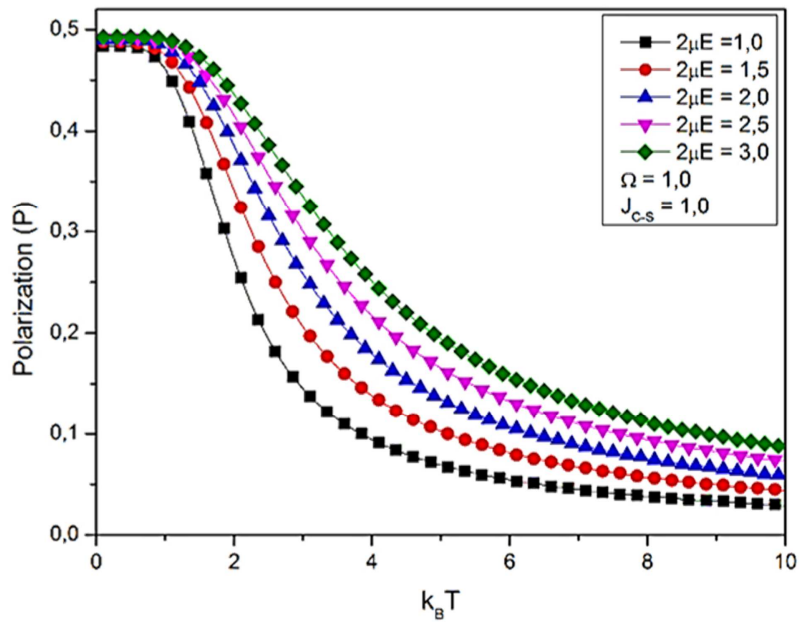

Figure 4. Polarization according to the evolution of the electric field (E).

Longitudinal Susceptibilities - The susceptibility is a physical parameter which characterizes the polarization created by the electric field. Moreover, the susceptibility gives the information about the critical temperature. In the Figure 5, one reduced the susceptibilities in the range where the peaks are more visible. Thus at low temperature, the augmentation of the exchange interaction $J_{c-s}$ show a superposition of the curves of susceptibility from 1.0 to 2.5 , but the important information observed are the peaks at the temperatures $\mathrm{k}_{\mathrm{B}}$. $\mathrm{T}=1.20,2.50$ and 3.78 which represent the critical temperatures already seen in the Figure 2 . In the Figure 6 , the enhancement of transverse field $(\Omega)$ comes along the diminution of the curves of susceptibility. But the higher peaks obtained at different temperatures represent the critical temperatures already observed in the Figure 3 define by $\mathrm{k}_{\mathrm{B}} . \mathrm{T}=1.20$ (for $\Omega=1.0$ ), $\mathrm{k}_{\mathrm{B}} . \mathrm{T}=1.08$ (for $\Omega=1.5$ ) and $\mathrm{k}_{\mathrm{B}}$. $\mathrm{T}=0.88$ (for $\Omega=2.0$ ). We didn't observe a peak which characterize a transition temperature because as we already knew from the figure $3, \mathrm{p}(\mathrm{T})=0$ for $\Omega=2.5$ and 3.0. In the Figure 7 , we didn't report a peak for the curves of susceptibility which defined the critical temperature because of the non-zero applied field reason why there are no peaks for the phase's transitions and the susceptibilities simply fellow an asymptote as already reported in the Figure 4.

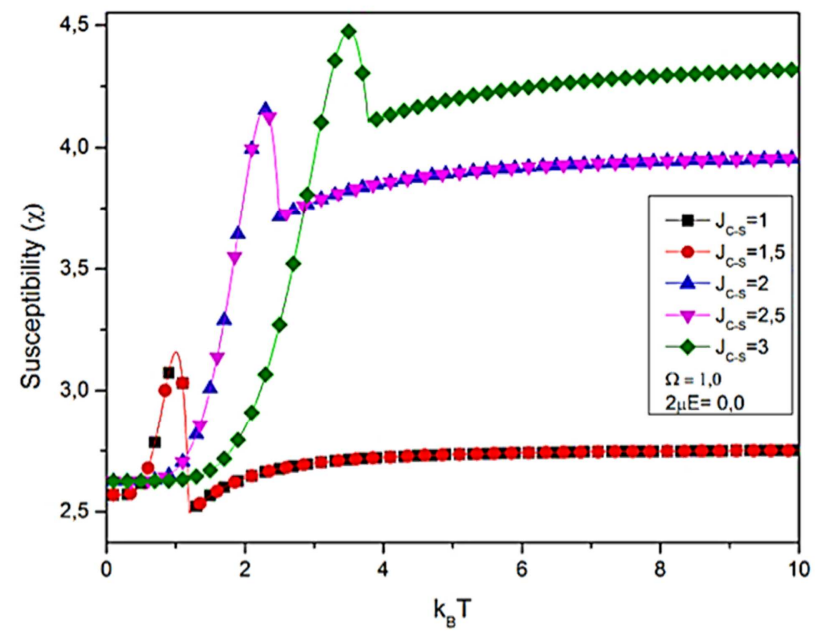

Figure 5. Susceptibility according to the evolution of the exchange interaction $\left(J_{C-S}\right)$.

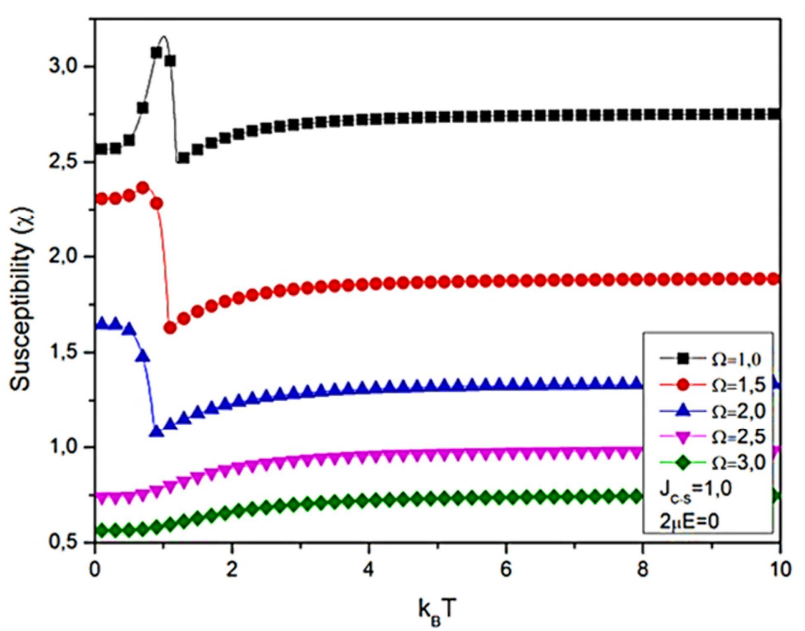

Figure 6. Susceptibility according to the evolution of the transverse field $(\Omega)$. 


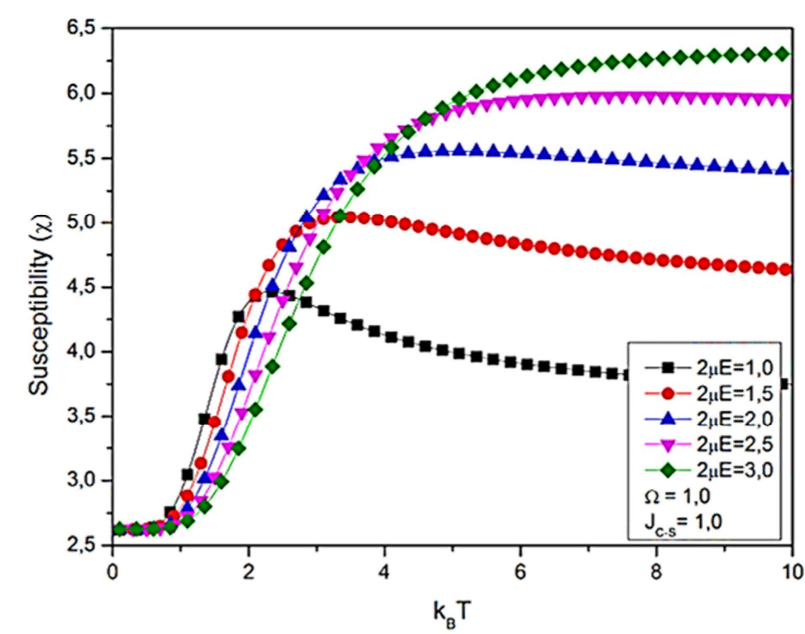

Figure 7. Susceptibility according to the evolution of the electric field (E).

Hysteresis - A ferroelectric material is characterized by a hysteresis loop which describes the polarization according to the electric field applied. With a Temperature $\mathrm{k}_{\mathrm{B}}$. $\mathrm{T}=1.0$ and a transverse field $\Omega=1.0$, one studied the influence of the electric field on the polarization. In the Figure 8 , one investigated the hysteresis with an enhancement of the exchange interaction, one reported that as the polarizations $\mathrm{p}$ (T) and susceptibilities $\chi(T)$, the increase of the exchange interaction provide a superposition of the hysteresis loop $\mathrm{p}$ (E) for certain values of $\mathrm{J}_{\mathrm{C}-\mathrm{S}}$. One noted the remanent polarization $\left(\mathrm{p}_{\mathrm{r}}\right)$ is 0.46 , the coercive field $\left(\mathrm{E}_{\mathrm{co}}\right)$ is 0.52 for $\mathrm{J}_{\mathrm{C} \text { - }}$ $\mathrm{s}=1.0,1.5$. For $\mathrm{J}_{\mathrm{C}-\mathrm{S}}=2.0,2.5$, the $\mathrm{p}_{\mathrm{r}}=0.49$ and $\mathrm{E}_{\mathrm{co}}=2.17$. From $\mathrm{J}_{\mathrm{C}-\mathrm{S}}=3.0$, the remanent polarization is $\mathrm{p}_{\mathrm{r}}=0.5$ and the coercive field $\mathrm{E}_{\mathrm{co}}=4.11$. One can see that the longitudinal polarizations curves are symmetric for both positive and negative longitudinal electric fields, and one observed the loops which characterize the ferroelectrics systems $\left(\mathrm{J}_{\mathrm{c}-\mathrm{s}}>0\right)$. In the Figure 9, one have the hysteresis for different values of the transverse field, with an enhancement of the transverse field $(\Omega)$, one has a decrease of the values of the polarization. For $\Omega=1.0$ one obtains a hysteresis characteristic of the ferroelectrics behaviors with a remanent polarization $\mathrm{p}_{\mathrm{r}}=0.46$ and the coercive electric field $E_{c o}=0.52$. For $\Omega=1.5$, the $p_{\mathrm{r}}$ is equal to 0.40 and $\mathrm{E}_{\mathrm{c}}=0.26$. And for $\Omega=2$, the $\mathrm{p}_{\mathrm{r}}=0.27$ and $\mathrm{E}_{\mathrm{c}}=0.06$. One lost the symmetric for the hysteresis from $\Omega=2.5$ to 3. One can interpret the phenomenon from $\Omega=2.5$ to 3 by the fact that the influence of the transverse field creates a disorder of the structure of the spins and makes a deformation of the unit cell. However, the remanent polarizations decrease with the increase of the transverse field. In the Figure 10, we already knew the temperatures create a perturbation on the unit cell. Therefore from a certain range of temperature $\mathrm{k}_{\mathrm{B}} . \mathrm{T}=1.0$ one is in the ferroelectric phase. Thus, one observe the normal shape of the hysteresis which characterize the ferroelectric phase with the remanent polarization $\mathrm{p}_{\mathrm{r}}=0.32$ and the coercive electric field $\mathrm{E}_{\mathrm{co}}=0.09$. From $\mathrm{k}_{\mathrm{B}} \mathrm{T}=1.5$ to 3.0 , the hysteresis loops become lines from the positive to the negative electric field and one can explain this phenomenon by the fact that these temperatures belong together in the para-electric phase $\mathrm{k}_{\mathrm{B}}$.
$\mathrm{T}_{\text {curie }}=1.2<\mathrm{k}_{\mathrm{B}} \mathrm{T}=1.5,2.0,2.5$ and 3.0 .

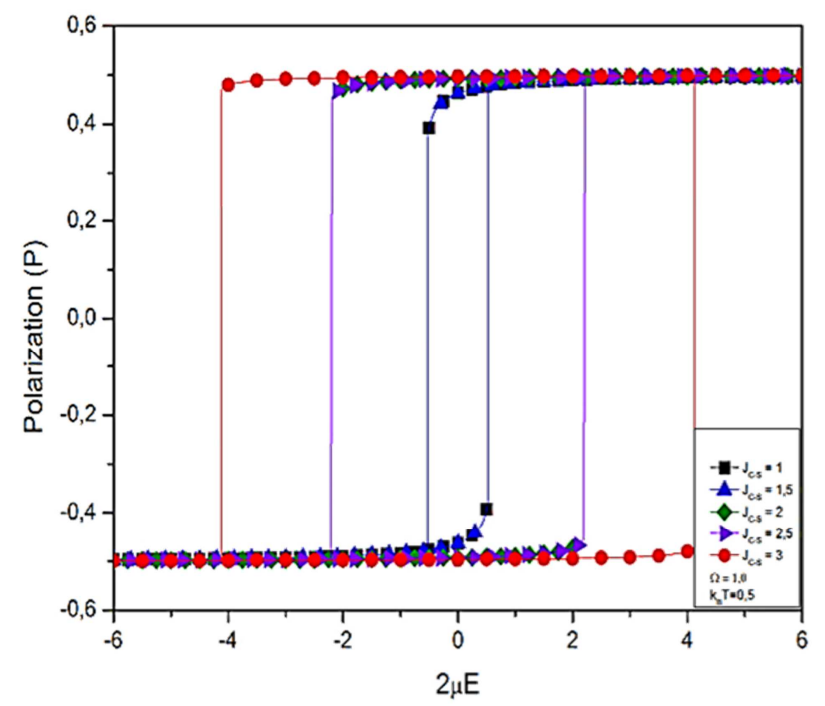

Figure 8. Hysteresis loop according to the evolution of the exchange interaction $\left(J_{C-S}\right)$.

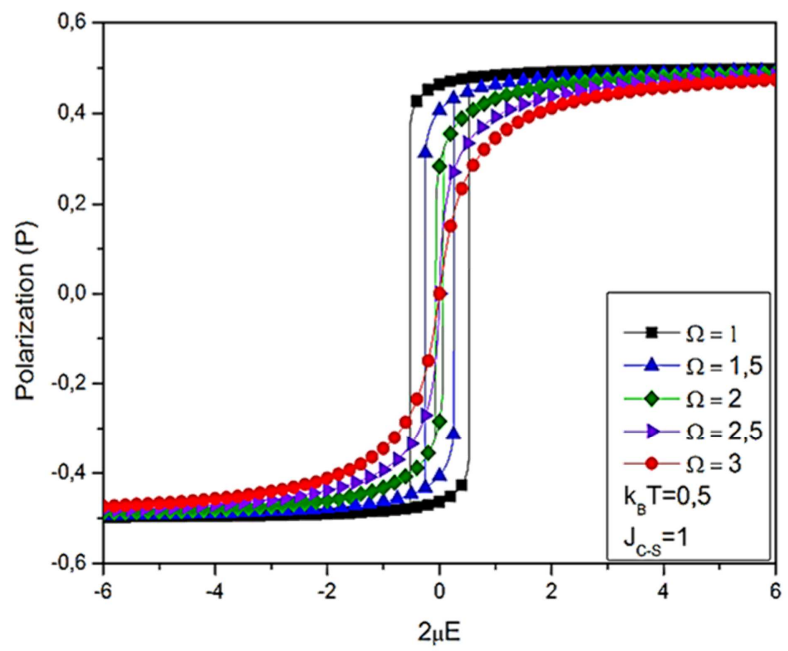

Figure 9. Hysteresis loop according to the evolution of the transverse field $(\Omega)$.

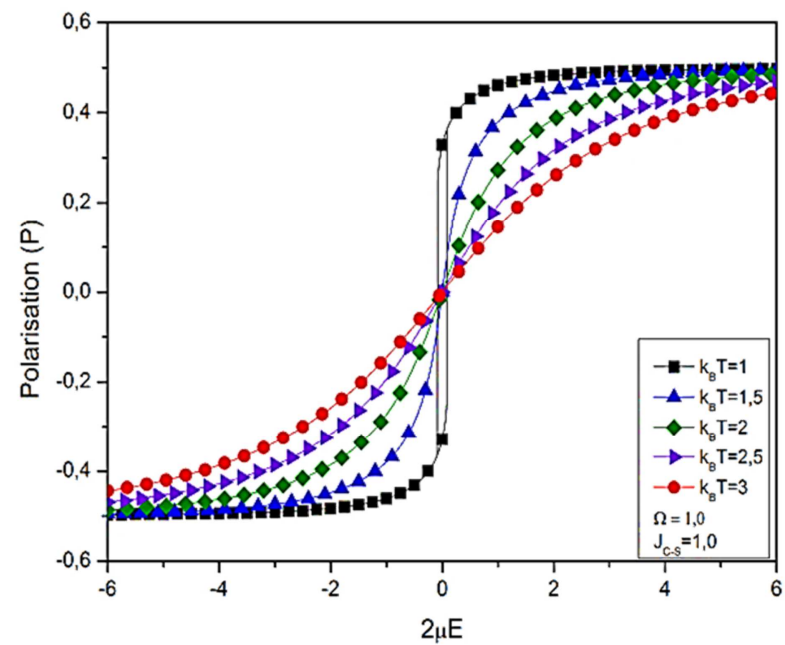

Figure 10. Hysteresis loop according to the evolution of the temperature (T). 
After the physical analyses above, the most important observation is the difference between the variation of the transverse field and the exchange interaction according to the polarizations. One noted that one can easily observe the maximum polarization with a weak value of the transverse field instead of the exchange interaction where the polarization becomes important with its augmentation. Benhouria and al. [40] observe the same behavior with the ferroelectrics cubic nanowires. And the authors [40] applied their investigation in the $(\mathrm{Ba}, \mathrm{Sr}) \mathrm{TiO}_{3}$ thin films [41]. One can explain this phenomenon by the fact that for a weak temperature, the spins don't fluctuate any more between the two equiprobable orientations of the weak polarization axis. However, like the axes of easy polarizations of the nanooctahedral structure are oriented by chance, the global polarization is null for a virgin sample. There remains possible, by the application of the transverse field, to provide enough energy to the system to overcome the barrier of anisotropy and align the spins according to the field. If during this time the field is brought back to zero, the spins do not have enough energy to pass again throughout the barrier of anisotropy. Thus the spins remain directed according to the balance position forming the weakest angle with the direction of the field.

\section{Conclusions}

One investigated the dielectrics properties of a nanooctahedral ferroelectric structure by the Transverse Ising Model (TIM). In this paper, we studied the influence of the exchange interaction, transverse field, temperature and electric field on the polarizations, susceptibilities and hysteresis loops. Moreover, from the unit cell of a structure it is easier to predict the physical properties than in a structure considered in large number. In another hand, one reported that between the exchange interaction and the transverse field under the influence of the temperature, the polarization can be well observed with a weak excitation of the transverse field instead of an increase of the exchange interaction. To finish, one shows the steps by using the transverse Ising model to model the ferroelectric system and with the same method one can investigate as well the ferromagnetic properties and even the other magnetic properties like antiferromagnetic or ferromagnetic. Otherwise, the method present in this paper will be used to investigate the random magnetics properties on the nanomaterials.

\section{References}

[1] R. Gopala Krishnan and S. Seshamma, Bull. Mater. Sci., Vol. 14, No. 6, December 1991, pp. 1349-1352.

[2] Alexander J. Hearmon, Federica Fabrizi, Laurent Ç. Chapon, R. D. Johnson, Dharmalingam Prabhakaran, Physical Review Letters, PRL 108, 237201 (2012).

[3] Martí Gich, Ignasi Fina, Alessio Morelli, Florencio Sánchez, Marin Alexe, Jaume Gàzquez, Josep Fontcuberta and Anna
Roig, Adv. Mater. 2014, 26: 4645-4652.

[4] Jaita Paul, Takeshi Nishimatsu, Yashiyuki Kawazoe, Umesh V. Waghmare, Physical Review Letters, PRL 99, 077601 (2007).

[5] Donna C. Arnold, Kevin S. Knight, Finlay D. Morrison, Philip Lightfoot, Physical Review Letters, PRL 102, 027602 (2009).

[6] Y. Park, Solid State Commun. 112 (1999) 167.

[7] E. K. Tan, J. Osman, D. R. Tilley, Solid State Commun. 117 (2001) 59.

[8] Y. Yamasaki, S. Miyasaka, Y. Kaneko, J. P. He, T. Arima, Y. Tokura, Physical Review Letters, PRL 96, 207204 (2006).

[9] K. T. Li, V. C. Lo, Solid State Commun. 132 (2004) 49.

[10] J. M. Wesselinowa, S. Trimper, Phys. Rev. B 69 (2004) 024105 .

[11] B. Teng, H. K. Sy, Phys. Rev. B 69 (2004) 104115.

[12] Kevin F. Garrity, Karin M. Rabe, David Vanderbilt, PhysicalReview Letters, PRL 112, 127601 (2014).

[13] T. Kaneyoshi, Phys. A 293 (2001) 200.

[14] T. Kaneyoshi, Phys. A 319 (2003) 355.

[15] Ruixiang Fei, Wei Kang, Li Yang, physical Review Letters, PRL 117, 097601 (2016).

[16] A. OubelKacem, I. Essaoudi, A. Ainane, M. Saber, J. Gonzalez, K. Barner, Ferroelectric films described by the transverse Ising model, Physica B 404 (2009) 4190-4197.

[17] P. G. de Gennes, Solid State Commun. 1 (1963) 132.

[18] R. Blinc, B. Zeks, Soft Modes in Ferroelectrics and Antiferroelectrics, North- Holland, Amsterdam, 1974.

[19] R. Pirc, R. Blinc, Phys. Rev. B 70 (2004) 134107.

[20] X. Z. Wang, X. Y. Jiao, J. J. Wang, J. Phys. Condens. Matter 4 (1992) 3651.

[21] Y. G. Wang, W. L. Zhong, P. L. Zhong, Phys. Rev. B 53 (1996) 11439.

[22] W. L. Zhong, B. D. Qu, P. L. Zhang, Y. G. Wang, Phys. Rev. B 50 (1994) 12375.

[23] H. X. Cao, Z. Y. Li, J. Phys. Condens. Matter 15 (2003) 6301.

[24] J. M. Wesselinowa, Phys. Stat. Sol. (b) 223 (2001) 737.

[25] C. L. Wang, W. L. Zhong, P. L. Zhang, J. Phys. Condens. Matter 3 (1992) 4749.

[26] C. L. Wang, S. R. P. Smith, D. R. Tilley, J. Phys. Condens. Matter 6 (1994) 9633.

[27] I. B. Misirlioglu, G. Akcay, S. Zhong, S. P. Alpay, J. Appl. Phys. 101 (2007) 036107.

[28] L. Q. Chen, in: C. H. Ahn, K. M. Rabe, J.-M. Triscone (Eds.), Physics of Ferroelectrics: A Modern Perspective, Springer, Berlin, 2007, pp. 363-372.

[29] Y. Watanabe, Phys. Rev. B 57 (1998) 789.

[30] L. W. Zhong, Y. G. Wang, P. L. Zhang, Phys. Lett. A 189 (1994) 121. 
[31] D. L. Tao, Y. Z. Wu, Z. Y. Li, Phys. Stat. Sol. (b) 231 (2002) 3.

[32] J. M. Wesselinowa, Phys. Stat. Sol. (b) 231 (2002) 187.

[33] K. lshikawa, K. Yoshikawa, N. Okada, Phys. Rev. B 37 (1988) 5852 .

[34] E. V. Colla, A. V. Fokin, Yu. A. Kumzerov, Solid State Commun. 103 (1997) 127.

[35] Alioune Aidara Diouf, Bassirou Lo, Alhadj Hisseine Issaka Ali, Aboubaker Chedikh Beye, American Journal of Nanomaterials, 2016, Vol. 4, No. 1, 1-7.
[36] N. Boccara, Phys. Lett 94 A, 185 (1963).

[37] F. Zernike, Physica (Ultrecht) 7, 565 (1940).

[38] J. W. Tucker, J. Phys. A: Math. Gen. 27 (1994) 659.

[39] Oscar Iglesias, Amilcar Labarta, Journal of Magnetism and Magnetic Materials 738, 290 (2005).

[40] Y. Benhouria, I. Essaoudi, A. Ainane, R. Ahuja, F. Dujardin, J. Supercond Nov Magn, 10.1007/s10948-014-2571-7 (2014).

[41] Y. Benhouria, I. Essaoudi, A. Ainane, R. Ahuja, F. Dujardin, Chinese Jornal of Physics, 10.1016/j.cjph.2016.06.012 (2016). 